\title{
Effect of endurance exercise training on morphological changes in rat heart tissue following experimental myocardial infarction
}

\author{
Akbar Azamian Jazi ${ }^{1}$, Hadi Abdi ${ }^{2 *}$, Mohammad Reza Haffezi Ahmadi ${ }^{3}$, Javad Cheraghi ${ }^{4}$
}

1. Department of Exercise Physiology, Faculty of Literature and Human Sciences, Shahrekord University, Shahrekord, Iran

2. Department of Physical Education, Payam-e-noor University, Tehran, Iran

3. Department of Pathobiology, Faculty of Medicine, Ilam University of Medical Science, Ilam, Iran

4. Department of Physiology, Faculty of Veterinary Medicine, Ilam University, Ilam, Iran

*Corresponding author:Tel: +98 9189422723 Fax: +98 8432221052

Address: Department of physical education, Payam-e-noor university, Tehran, Iran

E-mail: abdi_197866@yahoo.com

Received: 2015/05/24 revised: 2015/07/24 accepted: 2015/07/26

\section{Abstract}

Introduction: Cardiac remodeling after myocardial infarction, is associated with progressive ventricular dysfunction and cardiovascular death. The purpose of this study was to examine the effect of endurance exercise training on morphological changes in rat heart tissue following experimental myocardial infarction.

Materials and methods: Rats used in this experiment (8-10 weeks old, $235 \pm 5 \mathrm{~g}$ body weight) after homogenization according to body weight, were randomly divided to three groups: Healthy (Sham), control (Sedentary) and endurance training groups. Myocardial infarction was induced by subcutaneous injection of isoprenaline $(150 \mathrm{mg} / \mathrm{kg})$ in two consecutive days with an interval of 24 hours. Endurance training initiated 2 days after infarction and continued for 4 weeks. In order to assess the necrosis lesion and fibrosis tissue, Hematoxylin-Eosin and Masson's trichrome staining were used, respectively. Data were analyzed by using one-way analysis of variance (ANOVA) at $\mathrm{P}<0.05$ level.

Results: The results indicated that subcutaneous injection of isoprenaline, induced myocardial infarction in rat heart tissue. Rate of necrotic lesion in the Endurance training group was significantly higher than Sedentary (control) group $(\mathrm{P}=0.005)$. Also, the size of fibrosis tissue in the Endurance training group was significantly higher than the sedentary (control) group $(\mathrm{P}=0.001)$.

Conclusion: In general, results of this study showed that 4 weeks of endurance exercise training after myocardial infarction exacerbate tissue injury in rat heart.

Keywords: Endurance training, Myocardial infarction, Morphological changes, Rat

\section{Introduction}

One of the main causes of death is cardiovascular disease, which accounts for about $30 \%$ of all deaths in the world annually (1). Each year, about 17.1 million people in the world died due to cardiovascular diseases, of which about 7.2 million people, is caused by myocardial infarction (MI) (2). MI is the most common cause of heart failure (HF) and underlying left ventricular systolic and diastolic dysfunction that leads to increased mortality and a major impairment in quality of life (3). Cardiac structural changes after MI, consists of a series of changes in the left ventricle, which leads to a decrease in left ventricular function (4). Following MI, cardiac remodeling is associated with an inflammatory reaction, followed by scar formation at the site of infarction as well as changes in the non-infarcted myocardium, including interstitial fibrosis and vascular remodeling (5). 
After MI, cardiac remodeling is associated with progressive ventricular dysfunction and cardiovascular death (6). Current non pharmacological treatment of patients after MI, often includes exercise training as an element of cardiac rehabilitation (7). However, the results of research about the effects of exercise on post-MI cardiac morphological remodeling is contradictory. For example, Haykowsky et al. (2011) reported that exercise training after infarction (started 1 week after MI) improved LV remodeling (8). However, Batista et al. (2013), in assessment of effects of three-month endurance exercise training, which started 5 and 21 days after MI, reported that the infarct size was not different in experimental groups compared with control group (6). As well as, Jain et al. (2000) investigated the effect of 6 weeks endurance exercise training on infarct size in rats with MI. They reported that, the infarct size among the exercise and control groups has no significant difference, and this index was slightly higher in exercise group than control group. They also reported that exercise training, resulted in additional scar thinning (7). Xu et al. (2008) suggested that post-MI exercise training attenuate myocardial fibrosis and preserve post-MI cardiac function (9). In this context, De Waard et al. (2007) investigated the effect of 8 weeks of endurance exercise training, beginning 24 hours after MI in infarcted rats. They reported that this exercise protocol has no effect on survival rate, infarct size and LV volume (10). However, Gaudron et al. (1994) reported that endurance swim training, 4 and 21 days after coronary artery occlusion cause no LV remodeling in rats with small infarct, but in rats with large infarct, early endurance training aggravated the remodeling of $\mathrm{LV}$ and increased mortality (11). In study by Wan et al (2007), 8 weeks of running on a treadmill, 1 and 6 weeks after MI, independent of its inception, caused non-significant increase in the infarct size in exercise groups compared with sedentary control group and cardiac tissue remodeling were similar in both training protocol (12). Orenstein et al. (1995) reported that exercise training following MI in rats, can improve the adverse remodeling process by attenuating ventricular dilation and reducing wall tension (13). Serra et al. (2010) suggested that 13-weeks of endurance training in rats, prevents adverse remodeling of LV that was caused by the injection of isoprenaline (14). Jugdutt et al. (1988) claimed that 12week low-intensity exercise after MI may trigger remodeling of LV, expansion of MI, further scar thinning and reduction of ejection fraction (15).

In summary, many studies have been done with respect to the effects of endurance exercise training on cardiac remodeling after MI in animal and human subjects. However, results of previous studies are not uniform and in some studies exercise has beneficial effects, in some without effect and has harmful effects in other studies. Therefore, the aim of the present study is to investigate the effect of 4-week endurance exercise training on cardiac morphological changes in rats following experimental MI induced by subcutaneous (SC) injection of isoprenaline .

\section{Materials and methods}

Animals: In this experiment, 26 male Wistar rats, weighing 210-245 $\mathrm{g}$ and aged 8-10 weeks, were bought. Matching by weight and one week accustomed to the new conditions, followed by participation in a familiarizing program, running on a rattreadmill at $6 \mathrm{~m} / \mathrm{min}$, no gradient, for 10 minutes, once a day, for 5 consecutive days (16). To force the rat to run a $0.5 \mathrm{~mA}$ electric shock was used. They had free access to water and food and all of them were housed at constant temperature (22$25^{\circ} \mathrm{C}$ ) on a $12 \mathrm{~h} \mathrm{light/dark} \mathrm{cycle} \mathrm{and} \mathrm{relative}$ humidity $45-55 \%$ in plastic cages in the animal house of Ilam University.

MI Induction: Isoprenaline solution in normal saline was injected SC at a rate of $(150 \mathrm{mg} / \mathrm{kg})$ on two consecutive days with an interval of 24 hours for MI induction. 
Selection of injectable dose was based on a pilot study. In this case, the three doses of 100,150 and $200 \mathrm{mg} / \mathrm{kg}$ of body weight were used. Evaluating the rats cardiac tissue showed that MI was induced in doses of 150 and $200 \mathrm{mg} / \mathrm{kg}$. Therefore the dose of $150 \mathrm{mg} / \mathrm{kg}$ of body weight was selected. A total of 26 rats, 6 rats was allocated in the sham control group and received exclusively normal saline SC in two days. The remaining 20 rats received isoprenaline $\mathrm{SC}$ at a rate of $(150 \mathrm{mg} / \mathrm{kg})$ on two consecutive days. A total of 20 rats that had received isoprenaline, 4 (total 20\%) died after the second injection. To ensure infarction, 48 hours after the second injection 4 rats were killed and the remaining 12 rats were randomly assigned to one of the following two groups, control group $(n=6)$, and endurance training group $(n=6)$.

Study design and data collection: According to Figure 1, training intervention began two days after MI. Endurance training group, started the main training program at a speed of $10 \mathrm{~m} / \mathrm{min}$, for 10 minutes, once a day, for 5 sessions per week. The speed and duration of training were gradually increased to $16 \mathrm{~m} / \mathrm{min}$ and 50 minutes a day (including 5 min warm-up at $10 \mathrm{~m} / \mathrm{min}$ ) by the end of the second week, the intensity and duration maintained constant throughout the experiment. Endurance exercise protocol continued for
4 weeks. This exercise regimen intensity is average about $55 \%$ of maximal oxygen consumption $\left(\mathrm{VO}_{2} \max \right)$ and is well tolerated by infarcted rats $(9,17)$. In order to neutralize the effect of potential stress of the animals on the treadmill, healthy and control groups were placed on the device for 5 minutes once a day at a speed of 5 $\mathrm{m} / \mathrm{min}$ daily.

Determination of myocyte necrosis and fibrosis: To assess the tissue damage caused by isoprenaline, 48 hours after MI induction 4 rats were anesthetized with chloroform and under deep anesthesia, heart isolated and after weighing was placed in $10 \%$ formalin as a fixative. Hearts were cut longitudinally 24 hours later, and were immersed into molten paraffin. After preparation of paraffin blocks, using microtome $4 \mathrm{~mm}$ thick sections were prepared and mounted on glass slides. Each section was stained with HematoxylinEosin (H\&E) to determine myocyte necrosis $(\mathrm{MN})$. Also to assess the fibrous tissue (FT) Masson's trichrome staining was used. After staining, the samples were examined using an optical microscope Olympus and morphological changes were objectively quantified by a pathologist. Other rats were killed 24 hours after the last training session (28 days after MI) and diagnostic tests carried out as previous noted.

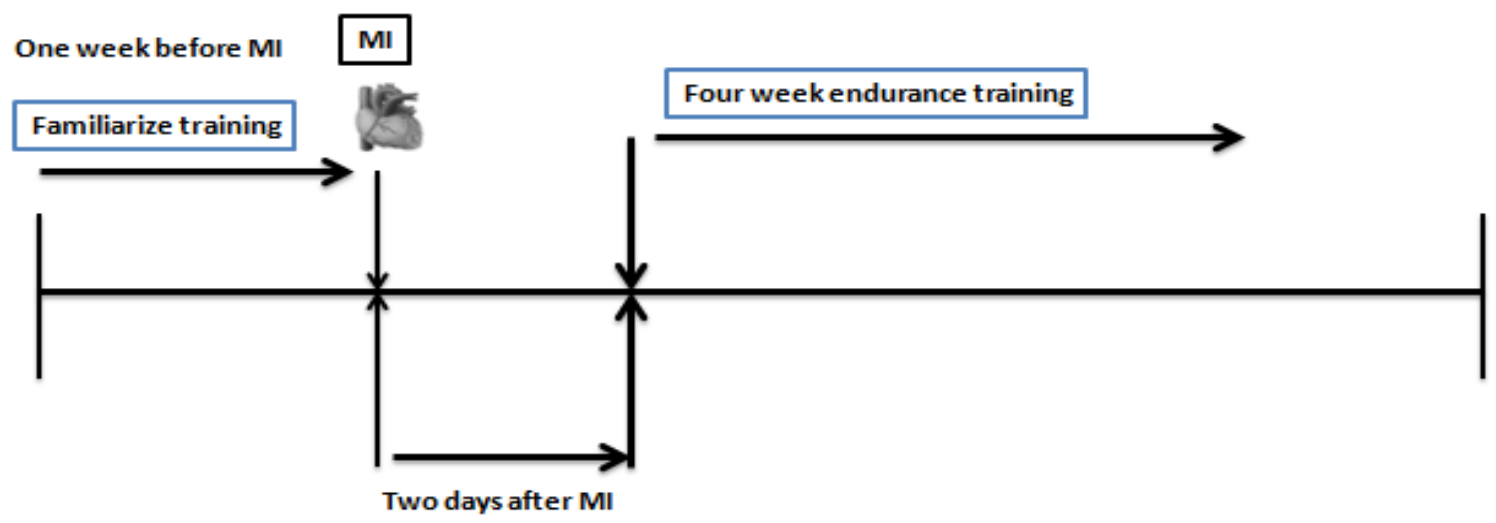

Figure 1. The outline of research design. 


\section{Statistical analysis}

For data analysis SPSS version 16, descriptive and inferential statistics were used. In the description level the mean and standard deviation, and in inferential level after confirming the normalization of data distribution by the Kolmogorov-Smirnov test, analysis of variance (ANOVA) and Scheffe post hoc test was used. The significant difference between groups, were considered $\mathrm{P}<0.05$.

\section{Results}

The results showed that $\mathrm{SC}$ injection of isoprenaline $(150 \mathrm{mg} / \mathrm{kg})$ on two consecutive days with an interval of 24 hours induced MI in male Wistar rats. $\mathrm{H} \&$ E staining showed that the rate of inflammatory cell and necrosis damage caused by isoprenaline ( 2 days after MI) was very severe, indicating the MI induction (Figure 1). There were no death during 4 weeks after MI in any of the groups.

Body weight, heart weight and heart weight/body weight ratio: The results of analysis of variance (ANOVA) test showed that among the indexes of body mass, heart weight, heart weight to body weight ratio, there were significant differences between groups of this study. Results of Scheffe post hoc test showed that there were no significant differences in body weight between endurance training group and the healthy group. But between these groups and the control group (sedentary) there is a significant difference $(\mathrm{P}=0.001)$. The difference in body weight between the control group and the values of the second day after infarction is not significant. Heart weight in MI groups (training and control) was not significantly different, but in endurance training group and healthy group was statistically significant $(\mathrm{P}<0.001)$. Heart weight to body weight ratio in $\mathrm{MI}$ groups (training and control) compared with the healthy group was significantly increased $(\mathrm{P}<0.05)$. However, the heart weight to body weight ratio was not significantly different between endurance training group and control group $(\mathrm{P}>0.05)$ (Table 1).

Necrosis damage and fibrosis tissue: In association with necrosis damage, the difference between endurance training and control group at $(\mathrm{p}=0.005)$ and between endurance training and the healthy group, as well as between control group and healthy group at $(\mathrm{p}=0.001)$ level were statistically significant (Figure 2 and table 1). The difference between mutually groups in relation to the percentage of the fibrous tissue at $(\mathrm{p}=0.001)$ level were significant (Figure 2 and Table 1).

Table 1. Mean \pm SD of body weight, heart weight, heart weight to body weight ratio, necrosis damage and fibrous tissue.

\begin{tabular}{|c|c|c|c|c|}
\hline $\mathrm{Pariables}_{\text {Var }}$ & MI+ training & MI + Sedentary & $\begin{array}{c}\text { Basic values } \\
\text { ( } 2 \text { days after } \mathrm{MI})\end{array}$ & Sham \\
\hline Body weight (g) & $278 \pm 3.76$ & $246 \pm 4.93$ & $241 \pm 2.75$ & $275 \pm 9.63$ \\
\hline Heart weight (g) & $1.28 \pm 0.18$ & $1.06 \pm 0.08$ & $1.13 \pm 0.15$ & $0.95 \pm 0.09$ \\
\hline Heart weight/Body weight (g/kg) & $4.62 \pm 0.63$ & $4.33 \pm 0.34$ & $4.70 \pm 0.66$ & $3.63 \pm 0.29$ \\
\hline Necrosis damage ( $\%$, percentage $)$ & $25 \pm 2.28$ & $21.33 \pm 1.03$ & $80.75 \pm 0.95$ & - \\
\hline Fibrous tissue (\%,percentage $)$ & $29.75 \pm 0.98$ & $20.83 \pm 0.75$ & $5.62 \pm 0.47$ & - \\
\hline
\end{tabular}

$\mathrm{MI}=$ myocardial infarction; $\mathrm{g}=$ gram; $\mathrm{Kg}=$ kilogram. 


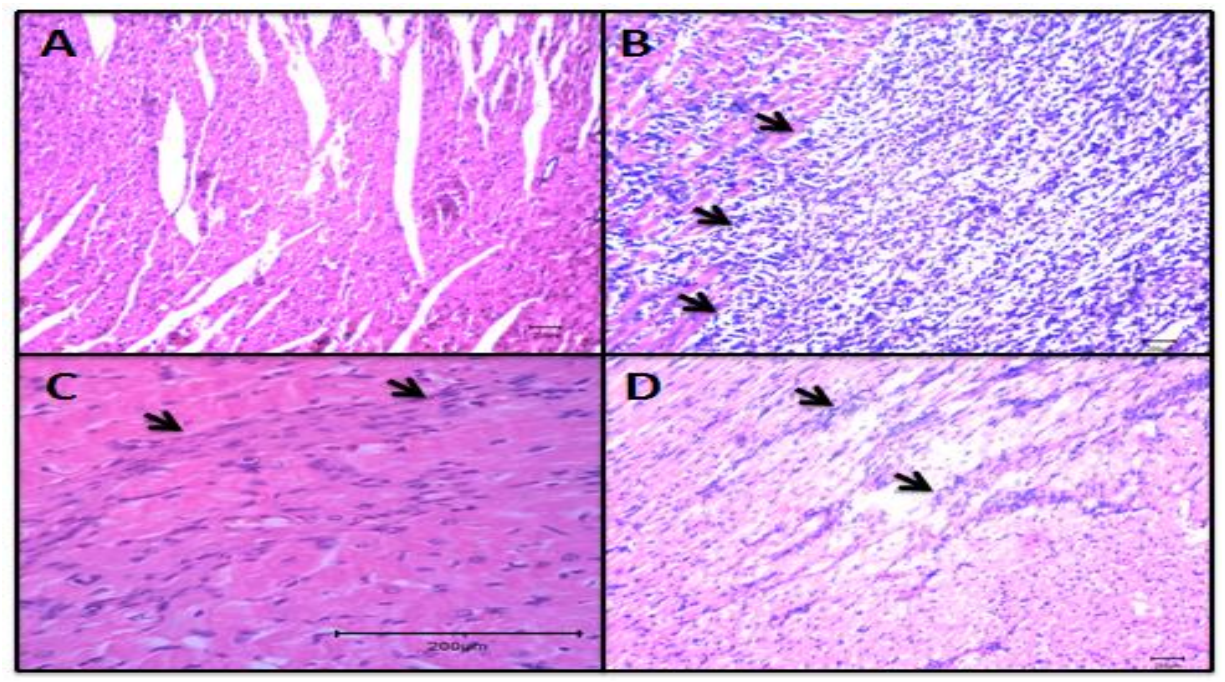

Figure 1. Representative images of histological sections stained with Hematoxylin \& Eosin of normal myocardial tissue in sham group (A) Sever necrosis in samples of 2 days after MI (B) Control group 28 days after MI (C) and endurance training group 28 days after MI (D). Areas marked by arrows show necrosis damage. Magnification: (C) 40x (A, B and D) 10x. Bar: $200 \mu \mathrm{m}$.

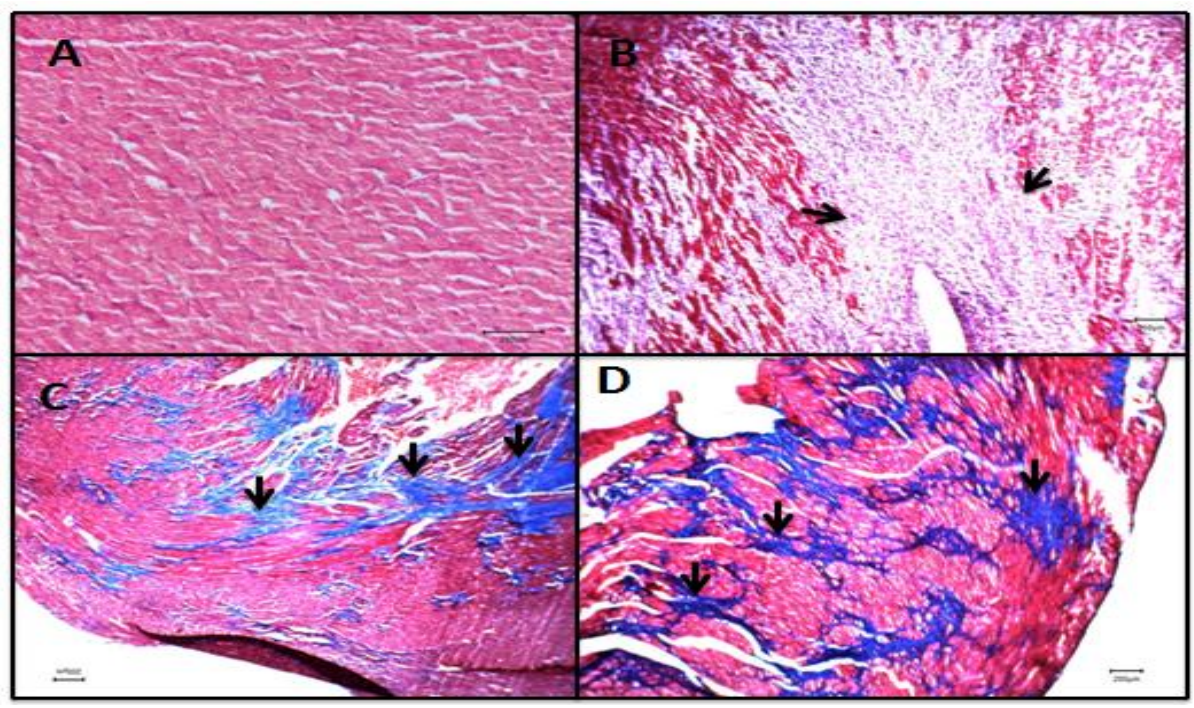

Figure 2. Representative images of histological sections stained with Masons-Trichrome of normal myocardial tissue in sham group (A) Sever fibrosis in samples of 2 days after MI (B) Control group 28 days after MI (C) and training group 28 days after MI (D) Areas marked by arrows show fibrous tissue. Magnification: (A) 40x (B, C and D) 10x. Bar: $200 \mu \mathrm{m}$.

\section{Discussion}

In the present study SC injection of isoprenaline $(150 \mathrm{mg} / \mathrm{kg})$ on two consecutive days, caused severe necrosis damage in cardiac tissue of rats that shows MI induction. The rate of cardiac hypertrophy in endurance training group compared with the control group had more increase. In other words, endurance training following infarction, resulted in enhancement of cardiac hypertrophy.

Previous studies have shown that endurance training after MI can have a beneficial effect $(9,13,14)$, without affecting $(6,7$, $10,12)$ or adverse effects $(11,15)$ on cardiac function and remodeling. The results of this study showed that endurance training running on a treadmill after MI 
increased the rate of necrosis damage and infarct size compared with the control group (no training). These results are consistent with results of Gaudron (1994) and Jugdutt (1998), but inconsistent with de Waard, $\mathrm{Xu}$, Serra and Orenstein results that reported the beneficial effects of exercise following MI. Several factors such as different experimental methods, the infarct size, type of activity, intensity and duration of activity may play a role in different effects of exercise following MI, as well as the onset time of exercise following MI may play an important role in cardiac remodeling after MI because inflammatory reactions caused by MI, fibrosis and scar formation occur at different stages after infarction (12). Therefore, from the reasons for inconsistent results of this investigation with above ones can be noted different methods of MI induction, duration of activity and the onset time of exercise following MI. In the present study endurance exercise training began 2 days after MI induction while in Orenstein and $\mathrm{Xu}$ studies began 5 weeks and 1 week after infarction respectively. In de Waard research although the exercise training same as present research began earlier after MI induction (24 hours after MI), but MI induction method was coronary artery occlusion and duration of activity was 8 weeks. Also, in Serra research that similar to the present study had used SC injection of isoproterenol to induce $\mathrm{MI}$, endurance exercise training began 12 weeks before isoproterenol injection and the beneficial effects of exercise in training group might be due to preconditioning effect of endurance exercise training. Some investigators have claimed that physical activity during the recovery phase (less than 1 week after MI) may act as wall stressor factor that increase the size of the LV (6). The key role in LV remodeling has been attributed to wall pressure of LV (11). In the early period of recovering after MI, overall remodeling of LV happens that includes extending the infarcted area, volume overload induced hypertrophy and cardiomyocytes destruction of noninfarcted area. It seems that cardiomyocyte hypertrophy following $\mathrm{MI}$ is due to pressure and volume overload that causes the increment in systolic and diastolic wall pressure, leading to increased cardiomyocyte diameter and length. Therefore exercising immediately after MI may lead to extension of the infarct size (13). It is reported that physical activity in patients with severe MI can be useful only when it is began later after infarction, as well as in animal studies, early exercise (less than one week) in mice after a moderate to severe MI may lead to adverse effects on LV remodeling (10). Thus, according to the severe necrosis damage that happened in infarction samples in this study, it is likely that the deleterious effects of endurance exercise training in this study is due to the severity of injury in the sample of the present study compared with previous studies. Investigations suggest that during the healing process, some of the factors may stimulate progressive cardiac dilation by increasing the ventricular wall pressure. As a result, it is clear that early exercise may act as a relative stressor factor (6). Increasing of wall pressure during exercise training is known as the reason of left ventricle dilation. It has been observed that the increase in the size of the LV is to be intensified after the exercise that began 3 weeks after MI, and is thought that early exercise after MI may be responsible for adverse LV remodeling (12). Exercise training immediately after MI, when necrosis and inflammation are high, may lead to increasing of $\mathrm{LV}$ pressure and thinning of infarcted wall that continues by collagen deposition in the thin wall, leading to LV remodeling (18). Thus, the deleterious effects of endurance exercise training after MI in the present study may be due to early exercise in contrast to previous studies.

Other reasons for inconsistent results of this study with previous researches can be noted the method of MI induction. In this study the SC injection of isoprenaline is used to 
induce MI, which causes damage as ischemia/reperfusion while in previous studies coronary artery occlusion of the left anterior-descending artery (LAD) is used. Isoprenaline effect mechanism in the cardiac damage, including the production of free radicals, increase in cytosolic calcium overload and mitochondria damage or dysfunction (19). Isoprenaline is a synthetic catecholamine and betaadrenergic receptor agonist that upon oxidation, generates highly toxic free radicals and stimulates membrane phospholipids peroxidation and severe myocardial membrane damage. It is reported that high levels of circulating catecholamines cause cardiac toxicity (19, 20). Isoprenaline or isoproterenol-induced myocardial injury represents the occurrence of oxidative damage, lipid peroxidation, LV hemodynamic and function changes following hypertrophy, inflammatory cells infiltration, fibrous and tissue necrosis. Isoprenaline-induced infarction, causes significant metabolical and morphological disorders in the heart muscle of laboratory animals, particularly rats, and shows biochemical and structural changes leading to cell damage and necrosis. Morphological changes observed in the coronary arteries may be justified by the mismatch between the oxygen supply and cardiac muscle demands after coronary hypotension and over activity of cardiac muscle result from isoprenaline (20).

Studies have shown that the capacity to secrete catecholamine in response to stimuli such as exercise, hypoglycemia and hypoxia is very high (21). In addition, it appears that physical activity increase the oxygen consumption 10 to 20 times in the body and enhance free radicals production in various tissues including the heart (22). It has been shown that oxidative stress caused by reactive oxygen species (ROS) plays a vital role in the pathogenesis of cardiac repair-remodeling following infarction (5). ROS in low concentrations serve as signaling molecules, but excess production of these agents causing adverse effects $(23,24)$. Oxidative stress occurs when ROS production is increase more than the antioxidant capacity. Following acute MI, oxidative stress expands in both infarcted and non-infarcted myocardium and expression of NADPH oxidase which the major source of oxygen anions $\left(\mathrm{O}_{2}^{-}\right)$is significantly increased in the infarcted myocardium (5). Repair/ remodeling process occurs following a loss of heart cells after MI. The process starts with the activation of matrix metalloproteinases (MMPs) that degenerate the extracellular matrix and structure of coronary artery (25). The proteolytic activity decreases at the end of the first week after MI along with increased expression of tissue inhibitor metalloproteinase (TIMPs). Proteolytic activity of metalloproteinases facilitates the migration of circulating inflammatory cells such as neutrophils, monocytes and macrophages to the infarct site. The inflammatory cells cause digestion and phagocytosis of the infarcted tissue. Inflammatory response reaches a peak within 1 to 2 weeks after MI and in 3 to 4 weeks after MI it will be reduced when inflammatory cells get cleaned from the infarct site (5). In addition, it is known that reperfusion after ischemia leads to increased production of ROS, mitochondrial permeability and cardiovascular death (26). Thus, according to the intolerance of exercise and oxygen uptake reduction $\left(\mathrm{VO}_{2}\right)$ in patients with $\mathrm{MI}$ (27), and also with regard to the incidence of hypoxia, coronary hypotension, cardiac hyperactivity and excessive oxidative stress, as well as reperfusion following isoprenaline-induced ischemia $(20,22,26)$, it seems that the accumulation of the effects of endurance exercise training and isoprenaline and stress of more dealing with electric shock sticks in training group rats, in the beginning of the exercise protocol in the present study creates more damage in cardiac tissue of endurance training group rats compared with the control group. Therefore, it is likely that the extent of damage in the endurance training group 
was more than the control group at the first. Training duration in the present study was 4 weeks, while in previous studies has been more than 4 weeks. Researches has shown that in order to achieve the maximum cardioprotection benefits of exercise and prevent adverse LV remodeling, post-MI patients should start aerobic exercise for about 1 week after discharge from the hospital and continue for longer duration (approx. 6 months) (8). Therefore, one of the reasons for inconsistent results of the

\section{References}

1. Lardizabal JA, Deedwania PC. Benefits of statin therapy and compliance in high risk cardiovascular patients. Vasc Health Risk Manag. 2010;6:843-53.

2. Organization WH. World health statistics 2010: World Health Organization; 2010.

3. Jorge L, Rodrigues B, Rosa KT, Malfitano C, Loureiro TC, Medeiros A, et al. Cardiac and peripheral adjustments induced by early exercise training intervention were associated with autonomic improvement in infarcted rats: role in functional capacity and mortality. Eur Heart J. 2011;32(7):904-12.

4. Chen CY, Hsu HC, Lee BC, Lin HJ, Chen YH, Huang HC, et al. Exercise training improves cardiac function in infarcted rabbits: involvement of autophagic function and fatty acid utilization. Eur J Heart Fail. 2010;12(4):323-30.

5. Sun Y. Myocardial repair/remodelling following infarction: roles of local factors. Cardiovasc Res. 2009;81(3):482-90.

6. Batista DF, Gonçalves AF, Rafacho BP, Santos PP, Minicucci MF, Azevedo PS, et al. Delayed rather than early exercise training attenuates ventricular remodeling after myocardial infarction. Int J Cardiol. 2013;170(1):e3-4.

7. Jain M, Liao R, Ngoy S, Whittaker P, Apstein CS, Eberli FR. Angiotensin II receptor blockade attenuates the present study with previous ones is probably less duration of training following MI.

\section{Conclusion}

Regarding the results of present study it appears that endurance exercise training following MI can act as a stress factor and cause deterioration of cardiac tissue damage and adverse cardiac remodeling.

deleterious effects of exercise training on post-MI ventricular remodelling in rats. Cardiovasc Res. 2000;46(1):66-72.

8. Haykowsky M, Scott J, Esch B, Schopflocher D, Myers J, Paterson I, et al. A meta-analysis of the effects of exercise training on left ventricular remodeling following myocardial infarction: start early and go longer for greatest exercise benefits on remodeling. Trials. 2011; 12:92.

9. Xu X, Wan W, Ji L, Lao S, Powers AS, Zhao $\mathrm{W}$, et al. Exercise training combined with angiotensin II receptor blockade limits post-infarct ventricular remodelling in rats. Cardiovasc Res. 2008;78(3):523-32.

10. de Waard MC, van der Velden J, Bito V, Ozdemir S, Biesmans L, Boontje $\mathrm{NM}$, et al. Early exercise training normalizes myofilament function and attenuates left ventricular pump dysfunction in mice with a large myocardial infarction. Circ Res. 2007;100(7):1079-88.

11. Gaudron $\mathrm{P}, \mathrm{Hu} \mathrm{K}$, Schamberger R, Budin M, Walter B, Ertl G. Effect of endurance training early or late after coronary artery occlusion on left ventricular remodeling, hemodynamics, and survival in rats with chronic transmural myocardial infarction. Circulation. 1994;89(1):402-12.

12. Wan W, Powers AS, Li J, Ji L, Erikson JM, Zhang JQ. Effect of postmyocardial infarction exercise training 
on the renin-angiotensin-aldosterone system and cardiac function. Am J Med Sci. 2007;334(4):265-73.

13. Orenstein TL, Parker TG, Butany JW, Goodman JM, Dawood F, Wen W-H, et al. Favorable left ventricular remodeling following large myocardial infarction by exercise training. Effect on ventricular morphology and gene expression. J Clin Invest. 1995;96(2):858-66.

14. Serra AJ, Santos MH, Bocalini DS, Antônio EL, Levy RF, Santos AA, et al. Exercise training inhibits inflammatory cytokines and more than prevents myocardial dysfunction in rats with sustained $\beta$-adrenergic hyperactivity. J Physiol. 2010;588(Pt 13):2431-42.

15. Jugdutt BI, Michorowski BL, Kappagoda CT. Exercise training after anterior Q wave myocardial infarction: importance of regional left ventricular function and topography. J Am Coll Cardiol. 1988;12(2):362-72.

16. Galvão TF, Matos $\mathrm{KC}$, Brum $\mathrm{PC}$, Negrão CE, Luz PLd, Chagas ACP. Cardioprotection conferred by exercise training is blunted by blockade of the opioid system. Clinics. 2011;66(1):1517.

17. Xu X, Zhao W, Lao S, Wilson BS, Erikson JM, Zhang JQ. Effects of exercise and L-arginine on ventricular remodeling and oxidative stress. Med Sci Sports Exerc. 2010;42(2):346-54.

18. Hammerman H, Schoen FJ, Kloner RA. Short-term exercise has a prolonged effect on scar formation after experimental acute myocardial infarction. J Am Coll Cardiol. 1983;2(5):979-82.

19. Zaafan MA, Zaki HF, El-Brairy AI, Kenawy SA. Protective effects of atorvastatin and quercetin on isoprenaline-induced myocardial infarction in rats. Bulletin of Faculty of
Pharmacy, Cairo University. 2013; 51(1):35-41.

20. Shukla SK, Sharma SB. $\beta$ Adrenoreceptor Agonist Isoproterenol Alters Oxidative Status, Inflammatory Signaling, Injury Markers and Apoptotic Cell Death in Myocardium of Rats. Indian J Clin Biochem. 2015;30(1):27-34.

21. Zouhal H, Jacob C, Delamarche P, Gratas-Delamarche A. Catecholamines and the effects of exercise, training and gender. Sports Med. 2008;38(5):40123.

22. Husain K, Hazelrigg SR. Oxidative injury due to chronic nitric oxide synthase inhibition in rat: effect of regular exercise on the heart. Biochim Biophys Acta. 2002;1587(1):75-82.

23. Sorescu D, Griendling KK. Reactive oxygen species, mitochondria, and NAD (P) $\mathrm{H}$ oxidases in the development and progression of heart failure. Congest Heart Fail. 2002;8(3):132-40.

24. Dhalla AK, Singal PK. Antioxidant changes in hypertrophied and failing guinea pig hearts. Am J Physiol. 1994;266(4 Pt 2):H1280-5.

25. Cleutjens JP, Kandala JC, Guarda E, Guntaka RV, Weber KT. Regulation of collagen degradation in the rat myocardium after infarction. J Mol Cell Cardiol. 1995;27(6):1281-92.

26. Chiong M, Wang Z, Pedrozo Z, Cao D, Troncoso $\mathrm{R}$, Ibacache $\mathrm{M}$, et al. Cardiomyocyte death: mechanisms and translational implications. Cell Death Dis. 2011;2:e244.

27. Guazzi M, Tumminello G, Reina G, Vicenzi M, Guazzi M. Atorvastatin therapy improves exercise oxygen uptake kinetics in post-myocardial infarction patients. Eur J Clin Invest. 2007;37(6):454-62. 